\title{
Comparison of Two Multipass Configurations for Scattered Light Amplification
}

\author{
M.J.R. Monteiro ${ }^{1}$, M. Machida ${ }^{1}$, A.M. Daltrini $^{1}$, and L.A. Berni ${ }^{2}$ \\ ${ }^{1}$ Instituto de Física "Gleb Wataghin", Universidade Estadual de Campinas \\ Caixa Postal 6165, 13083-970, Campinas, SP, Brazil \\ ${ }^{2}$ Associated Laboratory of Plasmas \\ Instituto Nacional de Pesquisas Espaciais \\ São José dos Campos, SP, Brazil
}

Received on 5 March, 2001. Revised version received on 8 May, 2001.

\begin{abstract}
Scattered light amplification by using multipass method is a very useful technique to increase Thomson and Rayleigh scattering signals for local plasma electron temperature and density measurements. Using Rayleigh scattering in nitrogen neutral gas chamber, the well-known technique of multipass system with two concentrical spherical mirrors is compared with new configuration by using two sets of lens and flat mirrors. The focusing capability of both configurations makes the difference to stray light and scattered signal related to spectrometer slit aperture and number of multipass. For the same number of laser beam pass and best slit aperture, the lens and plan mirrors new configuration presented lower stray light and higher scattered signal, although the gain of the usual two spherical mirrors still remains higher.
\end{abstract}

\section{Introduction}

Thomson scattering is often used to measure the local electron temperature, $[1,2]$ and electron density can be determined if calibration is done by use of Rayleigh scattering [3,4]. For this diagnostic method, one of the critical issues is the very low scattered light signal, which can be increased by use the of a higher laser power or by passing the laser beam many times through the same region in the plasma, known as multipass system [5-7]. Most commonly used configuration is the one with one lens and two concentrical spherical mirrors (CSM) [8-11]. This configuration has the main disadvantage of not allowing a well-defined spatial focal point region in the multipassage of the laser beam. Loosing therefore, not only spatial resolution but also well-defined solid angle for best collecting power spectrometer input.

In order to overcome these difficulties we propose a new configuration with use of two sets of lens and flat mirrors (LFM), which can define much better the focal point region, and therefore can provide a better spectrometer light collection capability.

Although the main purpose of this study is the increase of signal in the Thomson scattering diagnostic, we have performed the comparison using Rayleigh scat- tering on nitrogen neutral gas. At first because of the easiness and reproducibility, and secondly, because Rayleigh scattering is used to calibrate Thomson scattering density measurements. In our case, we have built a dummy sector of our tokamak [12], in order to have the same scattering geometry.

The relationship between Thomson and Rayleigh scattering can be seen by the following equations. In the measurements to determine the Thomson scattering profile, it is avoided the central region of the spectrum due to the strong influence of the stray-light. Therefore, the signal measured by a detector placed in the $N$-th range of the wavelength to be measured, with mean wavelength $\lambda_{N}$, in a solid angle $\Omega$, is [13-15]

$$
\begin{gathered}
S_{N}\left(\lambda_{N}\right)=R_{N} \cdot B_{S} \cdot \frac{P_{i} \cdot r_{0}^{2} \cdot c \cdot n_{e} \cdot L_{S} \cdot \Omega \cdot \eta \cdot G \cdot \tau}{2 \cdot \sqrt{\pi} \cdot \lambda_{i} \cdot \operatorname{sen}(\theta / 2)} . \\
\left(1-\frac{7 \cdot\left(\lambda_{N}-\lambda_{i}\right)}{2 \cdot \lambda_{i}}\right) \cdot \frac{1}{a} \cdot\left|\frac{1}{\epsilon}\right|^{2} \cdot \\
\exp \left(-\left(\frac{c^{2} \cdot\left(\lambda_{N}-\lambda_{i}\right)^{2}}{4 \cdot a^{2} \cdot \lambda_{i}^{2} \cdot \operatorname{sen}^{2}(\theta / 2) \cdot\left(1+\frac{\left(\lambda_{N}-\lambda_{i}\right)}{\lambda_{i}}\right)}\right)\right)
\end{gathered}
$$

where $P_{i}$ is the incident power, $R_{N}$ is the calibration of the photo-multiplier in the $N$-th range of the wavelength to be measured, $B_{S}$ is the aperture of the exit 
slit, $\theta$ is the scattered angle, $r_{0}^{2}=\left(d \sigma_{T} / d \Omega\right)$ is the classical electron radius for $90^{\circ}$ scattering, $c$ is light velocity, $L_{S}$ is the length of the scattering region, $\eta$ and $G$ are, respectively, the quantum efficiency and the gain of the photo-multiplier, $\tau$ is the transmission of the spectrometer, $\epsilon$ is the longitudinal dielectric function, and, finally, $a=\left(2 \cdot k \cdot T_{e} / m_{e}\right)^{1 / 2}$ is the electron thermal velocity. On the other hand, the Rayleigh signal measured by the photomultiplier, integrated in the solid angle of observation $\Omega$, is $[16]$ :

$$
S_{\text {Ray }}=P_{i} \cdot\left(\frac{d \sigma_{R}}{d \Omega}\right)_{\Omega} \cdot n_{R} \cdot L_{S} \cdot \Omega \cdot \eta \cdot G \cdot \tau
$$

or

$$
L_{S} \cdot \Omega=\frac{S_{\text {Ray }}}{P_{i} \cdot n_{R} \cdot\left(\frac{d \sigma_{R}}{d \Omega}\right)_{\Omega} \cdot \eta \cdot G \cdot \tau}
$$

Substituting eq.(2) into eq.(1), we obtain:

$$
\begin{gathered}
S_{N}\left(\lambda_{N}\right)=R_{N} \cdot B_{S} \cdot \frac{c \cdot n_{e}}{2 \cdot \sqrt{\pi} \cdot \lambda_{i} \cdot \operatorname{sen}(\theta / 2)} \\
\frac{S_{\text {Ray }}}{n_{R}} \cdot \frac{d \sigma_{T} / d \Omega}{d \sigma_{R} / d \Omega} \cdot\left(1-\frac{7 \cdot\left(\lambda_{N}-\lambda_{i}\right)}{2 \cdot \lambda_{i}}\right) \cdot \frac{1}{a} \cdot\left|\frac{1}{\epsilon}\right|^{2} . \\
\exp \left(-\left(\frac{c^{2} \cdot\left(\lambda_{N}-\lambda_{i}\right)^{2}}{4 \cdot a^{2} \cdot \lambda_{i}^{2} \cdot \operatorname{sen}^{2}(\theta / 2) \cdot\left(1+\frac{\left(\lambda_{N}-\lambda_{i}\right)}{\lambda_{i}}\right)}\right)\right)
\end{gathered}
$$

where $d \sigma_{R} / d \Omega$ is the differential cross section of the gas molecules, $n_{R}$ is the density of the gas molecules, nitrogen in our case.

For eq.(3), the ratio $S_{\text {Ray }} / n_{R}$ is given by the inclinatin of the calibration curve obtained from Rayleigh scattering, and the ratio between the Thomson and Rayleigh cross sections is given elsewhere [15].

\section{Experimental set up}

The experimental set up with dummy scattering vessel is shown in Fig. 1. The scattering vessel was built exactly with the same geometrical parameters of the tokamak NOVA-UNICAMP.

The ruby laser in $Q$-switched mode, and an energy pulse of 3J in $40 \mathrm{~ns}$ has beam divergence of $10^{-3} \mathrm{rad}$. After many passages of laser beam through the central region, it is collected in a beam dump.

For the alignment, we used two He-Ne lasers, one to align the ruby laser and the other to align the spectrometer GCA/McPHERSON-2051 with a fotomultiplier FOR EMI TUBE 9558 QB.

The beams from both He-Ne lasers should cross at the center of the vessel, which is defined as the scattering region. Notice that a photodiode is used as monitor for the ruby laser, by taking the first reflection from the prism, and for scattered light collection two lenses are used with the spectrometer to have a maximum solid angle match.

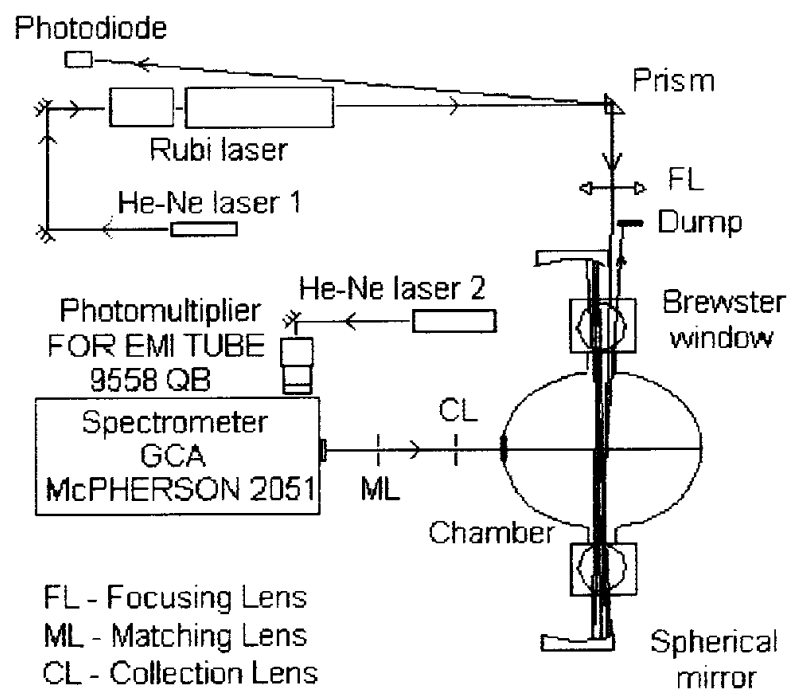

Figure 1. Overview of experimental set up for two spherical mirrors and lens.

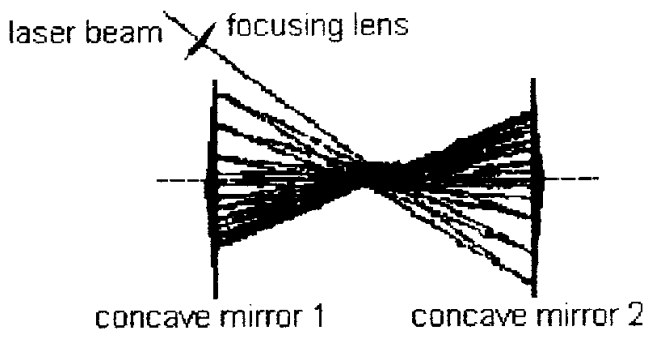

a) CSM system.

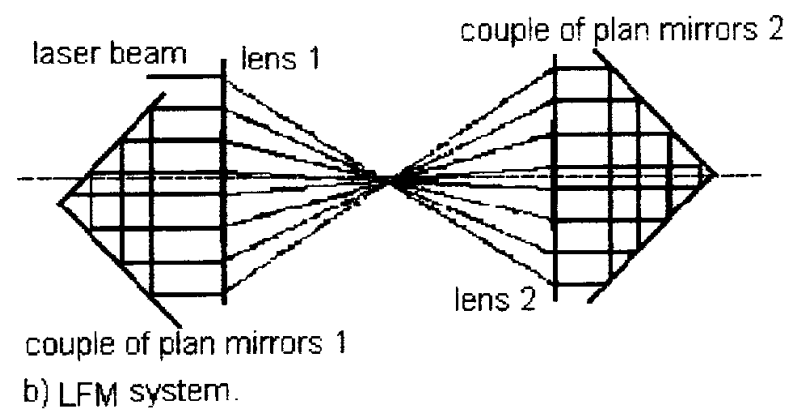

Figure 2: a) Concentrically spherical mirrors with lens configuration, CSM system, and b) Lens and flat mirrors configuration, LFM system. Notice the focusing effect at the lower picture.

In Fig. 2-a) it is shown the concentrical spherical mirror configuration. CSM, which consists of two equal spherical mirros, with diameter of $6.0 \mathrm{~cm}$ and focal distance of $12.5 \mathrm{~cm}$, and one focusing lens set outside Roland circle, with diameter of $4.5 \mathrm{~cm}$ and focal distance of $37.0 \mathrm{~cm}$. The maximum number of multipass 
is defined basically by the beam diameter and the distance between the edge of the spherical mirror and the beam entrance position, and it can provide a very high number of passages.

In Fig. 2-b) it is shown the proposed lens and flat mirrors configuration, LFM, which consists of two lenses, with diameter of $6.0 \mathrm{~cm}$ and focal distance of $25.0 \mathrm{~cm}$, and a couple of 90-degree plane mirrors, with $4.0 \mathrm{~cm}$ of width and $5.0 \mathrm{~cm}$ length. The maximum number of multipass is defined by the ratio between lens and beam diameter, in our case $N=6$.

As can be seen, CSM presents a very high number of passages but a poor focal region, which makes the alignment with the spectometer more difficult and causes the loss of the captured scattered signals. On the other hand, the LFM presents an excellent concentration of the laser beam, although it has the disvantage of a lower number of passage capability. To increase the number of passages one should have smaller diameter of the input beam and larger diameter lens and mirrors. In our case, we have used basically same sized optical components for both configurations for a comparison, and set the number of passages up to six.

\section{Results}

The typical single pass Rayleigh scattering and monitor signal are shown in Fig. 3. The gain of system is defined as the ratio between the normalized scattered signal (Rayleigh scattered light/laser monitor) of the single pass and the multipass signal. Normalization by area and peak value showed no difference for our calculations.

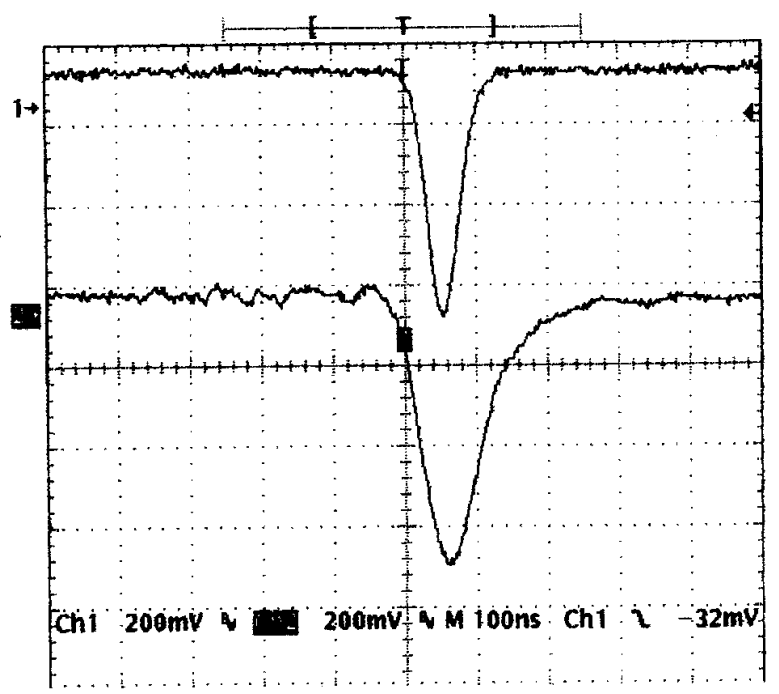

Figure 3. Characteristic signal for single pass mode, above is laser monitor; and lower is the signal for Rayleigh scattered light, 100ns/div.

The effect of stray light in the scattered signal is a very important issue to be considered, since it defines the lower limit of the temperature measurements.
In each case, the stray light was obtained by pumping down the chamber up to $10^{-3}$ Torr, and for scattering measurements, the $N$ gas was filled in to desired pressure and left in rest for about one hour.

The influence of the spectrometer slit aperture on the scattered signal is very important, since it controls not only the amount of stray light getting into spectrometer but also solid angle matching between scattered region and spectrometer. We define as scattered signal the total signal taken in the $\mathrm{N}$ gas scattering less the stray light signal.

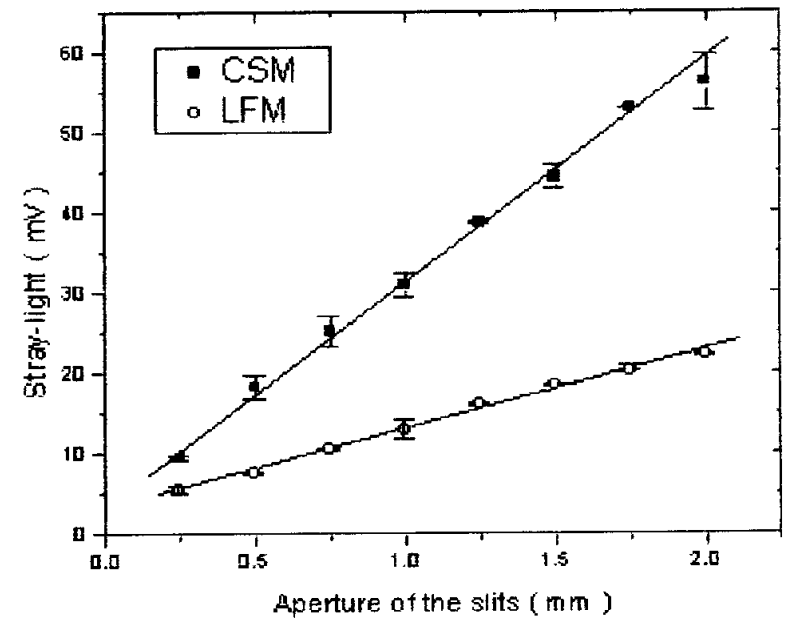

Figure 4. Stray light variation according to spectrometer slit aperture with $N=6$.

From Fig. 4, for six passages of the laser beam, we can see by the sloop of two configurations that CSM is much more sensitive to stray light than LFM.

Other interesting fact is the variation of the scattered light signal by spectrometer slit aperture. As shown by Fig. 5, we notice a saturation effect for CSM from slit aperture of $1.5 \mathrm{~mm}$, whereas for the LFM this is not seen, and is defined by the spectrometer maximum slit aperture of $2 \mathrm{~mm}$.

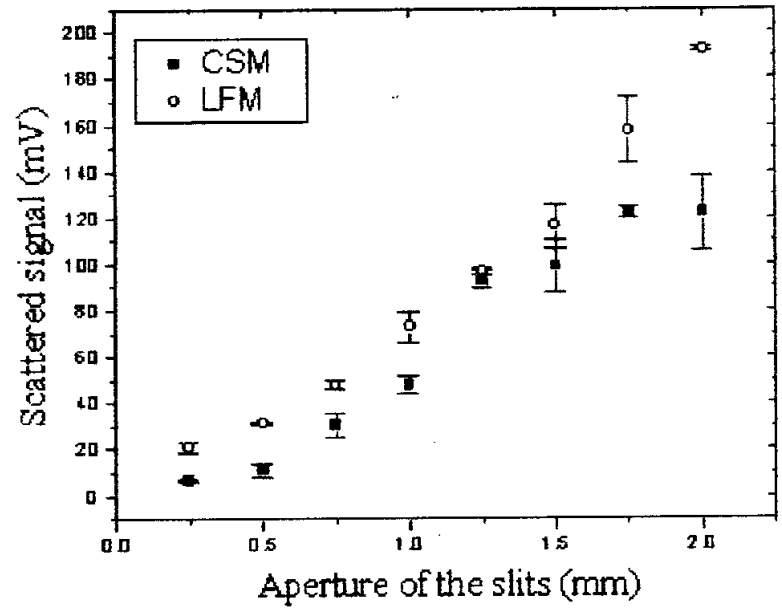

Figure 5. Scattered light variation related to spectrometer slit aperture. 
These effects are related to the focusing capability of the two configurations and should be more severe, with an increase of the number of passages.

By fixing now is the best slit aperture for CSM and LFM configurations the values of $1.5 \mathrm{~mm}$ and $2.0 \mathrm{~mm}$ respectively, we have studied the stray and scattered light related to the number of passages.

As can be seen on Fig. 6, the stray light is much stronger for CSM configuration, mainly for a higher pass number. This increase can also be seen on Fig. 4 if we take the $N=6$ and proper slit aperture.

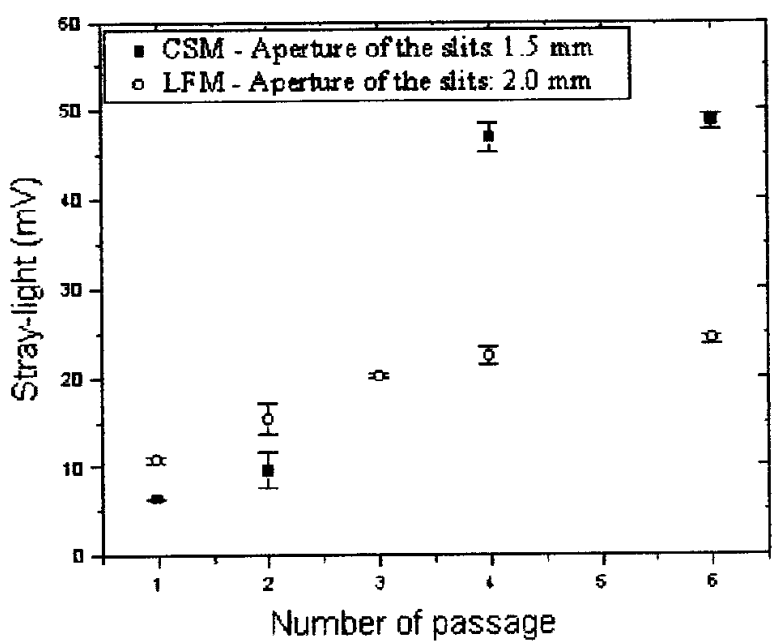

Figure 6. Stray light variation according to passage number using best-slit aperture condition for each configuration.

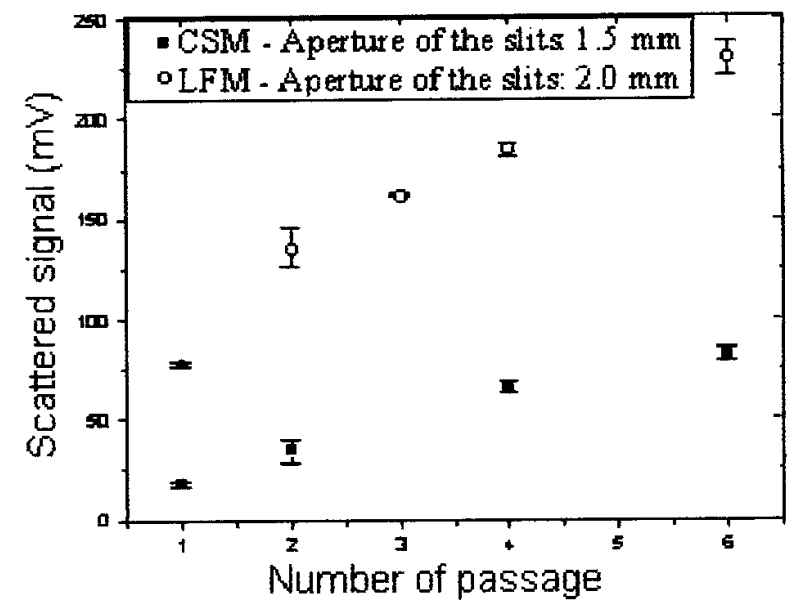

Figure 7. Scattered signal variation against number of passage using best spectrometer slit aperture for each configuration.

According to the variation of scattered signal related to number of passages, Fig. 7, we notice a much higher signal for LFM configuration. Since the focal point region is very well defined and matching of solid angle is also better for LFM, these results seen to be resonable, except for the fact that the LFM system had a small gain, $2.96 \pm 0.11$, if compared to the CSM system, $4.61 \pm 0.42$, for $N=6$.
We belive that the cause was mainly the loss of energy on the edges of the lens used in the LFM system, the anti-reflection coating for normal incidence on the lens edges seem to cause up to $10 \%$ loss of input energy pulse. But more precise studies are necessary to have better understanding.

\section{Conclusions}

The well-defined focusing region and therefore a better optical matching between scattered region and spectrometer etendue for two types of multipass systems have been analyzed. For the same number of passages LFM presented higher scattered light, lower stray light and no saturation of scattered light signal related to slit aperture due to its higher focusing capability. The CSM system can provide a higher number of passages, but it is also known that there is a limit on pass number to amplify the scattered signal.

Optical assembly of CSM is easier than LFM due to fewer components utilized on the CSM system. Nevertheless, optical alignment and matching with spectrometer in the LFM is much easier, since the focal point region is better defined.

Therefore, if enough space for bigger diameter lenses and mirrors exists, the lens and plane mirror configuration can give higher scattered signal and lower stray light for Thomson scattering diagnostics.

A comparison between these two configuratioins is now under investigation, using Hydrogen plasma in our tokamak NOVA-UNICAMP.

\section{Acknowledgments}

We would like to acknowledge Prof. Dr. Masayuki Fukao for his efforts to bring NOVA tokamak to our Lab., and for his precious and continuous help in the research conduced.

We are indebted to Dr. Mario Ueda - LAP - INPE for useful comments and corrections.

Author M.J.R. Monteiro acknowledges the fellowships support from FAPESP.

This work is supported by FAPESP - Fundação de Amparo à Pesquisa do Estado de São Paulo, FINEP, CNPq and CAPES.

\section{References}

[1] M. Bassan, R. Bilato, L. Giudicotti, R. Pasqualoto, and A. Sardella; Ver. Sci. Instrum. 68, 718 (1997).

[2] A. Côte, D. Michaud, N. Richard, R. Neufeld, and C. Legros; Ver. Sci. Instrum. 66, 487 (1995).

[3] L.A. Berni, D.O. Campos, M. Machida, S.A. Moshkalyov and S.V. Lebedev; Brazilian Journal of Physics, 26, 755 (1996). 
[4] D.G. Nilson, D.N. Hill, J.C. Evans, T.N. Calrstrom, C.L. Hsieh and R.E. Stockdale; Ver. Sci. Instrum. 70, 704 (1997).

[5] V.N. Bocharov and S.D. Kravchenko; Plasma Physics Reports, 20, 50 (1994).

[6] C.J. Barth, C.C. Chu, A.J.H. Donné; Ver. Sci. Instrum. 66, 501 (1995)

[7] R. Behn, J.H. Rommers, R.A. Pitts, Z.A. Pietrzyk, R. Chavan and B. Marletaz; Ver. Sci. Instrum. 70, 780 (1999).

[8] M.Y. Kantor and D. V. Kouprienko; Ver. Sci. Instrum. 70, 780 (1999).

[9] M.Y. Kantor; Technical Physics Letters, 23, 671 (1997).

[10] M.Y. Kantor and D.V. Kouprienko; Technical Physics Letters, 23, 321 (1997).
[11] M.Y. Kantor; Technical Physics Letters, 25, 860 (1999).

[12] M. Fukao, K. Mori, T. Tanihara; Plasma Phys. Control. Fusion, 33, 199 (1991).

[13] J. Shiffield; Plasma Scattering of Eletromagnetic Radiation, Academic Press, New York, (1975).

[14] L.A. Berni, "Espalhamento Thomson no Toróide Compacto TC - 1"; Ph.D. thesis, IFGW, UNICAMP - (1996).

[15] H.R. Grien, R.H. Lovberg; Methods of Experimental Physics; Academic Press, New York, (1970).

[16] J.D. Jackson; Classical Electrodynamics; John Wiley \& Sons, inc., New York, (1983). 